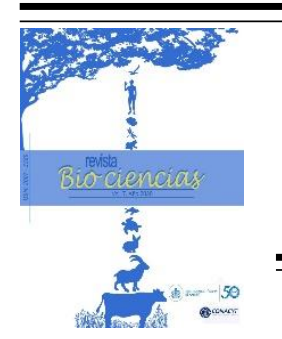

Revista Bio Ciencias

https://revistabiociencias.uan.edu.mx

ISSN 2007-3380

Memorias del 3er

Coloquio de

Nutrigenómica y

Biotecnología Acuícola

2020

Resumen en extenso

\title{
Análisis bioeconómico del cultivo intensivo foto-heterotrófico de camarón blanco (Litopenaeus vannamei) en alta salinidad con reposición mínima de agua, para el ciclo verano-otoño.
}

\author{
Moreno-Figueroa, L. D. *, Villarreal-Colmenares, H., Hernández-Llamas, A., Naranjo-Páramo, J., \\ Vargas-Mendieta, M., Hernández-Gurrola, J.A.
}

Centro de Investigaciones Biológicas del Noroeste, S.C. Calle Instituto Politécnico Nacional \# 195, La Paz, B.C.S. 23096, México.

*E-mail: morenofidaniel@gmail.com

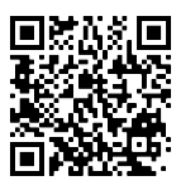

\begin{abstract}
Cite this paper/Como citar este artículo: Moreno-Figueroa, L. D., Villarreal-Colmenares, H., Hernández-Llamas, A., Naranjo-Páramo, J., Vargas-Mendieta, M., Hernández-Gurrola, J.A (2021). Análisis bioeconómico del cultivo intensivo foto-heterotrófico de camarón blanco (Litopenaeus vannamei) en alta salinidad con reposición mínima de agua, para el ciclo verano-otoño. Revista Bio Ciencias 8: (Suppl) Memorias del 3er Coloquio de Nutrigenómica y Biotecnología Acuícola 2020 (CONYBA) e1101. http://doi.org/10.15741/revbio.08Suppl.e1101
\end{abstract}

\section{Resumen}

Se desarrolló un análisis bioeconómico para inferir la producción y rendimiento económico del camarón blanco (Litopenaeus vannamei) en un sistema intensivo foto-heterotrófico en alta salinidad con reposición mínima de agua para el ciclo verano-otoño. La salinidad fue mantenida a $46( \pm 2.5 \mathrm{~g} / \mathrm{L})$, oxígeno disuelto a $4.4( \pm 0.4 \mathrm{mg} / \mathrm{L})$, $\mathrm{pH}$ a $7.8( \pm 0.2)$ y temperatura a $31.6\left( \pm 0.7^{\circ} \mathrm{C}\right)$. El análisis arrojó que el peso individual final promedio (wf) fue de $18.14 \mathrm{~g}$, la sobrevivencia promedio fue de $81.4 \%$ y una biomasa final promedio de $17.73 \mathrm{Ton} / \mathrm{ha}$, valores muy cercanos a los registrados en la base de datos (18.6 g para el peso final, $81.2 \%$ para la sobrevivencia y 18.1 Ton/ha para la biomasa final) y una utilidad neta promedio de $\$ 693,730$ pesos/Ha en 92 días de cultivo. El análisis de sensibilidad mostró que el precio de camarón fue el factor que más influenció la utilidad neta, seguido del peso final, concentración de oxígeno disuelto y temperatura. El cultivo intensivo foto-heterotrófico en alta salinidad es una innovadora tecnología que permite obtener elevados rendimientos de producción de camarón de manera biosegura. Los resultados económicos indican un alto potencial de aplicación y la conveniencia de continuar la investigación para su escalamiento.

\section{Abstract}

A bioeconomic analysis was developed to infer the production and economic performance of white shrimp (Litopenaeus vannamei) reared in a photo- heterotrophic, hypersaline intensive system with minimal water replacement for the summerautumn production cycle. Salinity was maintained at $46( \pm 2.5 \mathrm{~g} / \mathrm{L})$, dissolved oxygen at $4.4( \pm 0.4$ $\mathrm{mg} / \mathrm{L}), \mathrm{PH}$ at $7.8( \pm 0.2)$ and temperature at 31.6 $( \pm 0.7 \circ \mathrm{C})$. The analysis showed that final individual weight (wf) was $18.14 \mathrm{~g}$, survival was $81.4 \%$ and final biomass of 17.73 Ton / ha, values very close to those registered in the database (18.6 $\mathrm{g}$ for the final weight, $81.2 \%$ for survival and 18.1 Ton / ha for final biomass) and a net profit of $\$ 693,730$ mexican pesos / $\mathrm{Ha}$ in 92 days of rearing. The sensitivity analysis showed that the price of shrimp was the factor that most influenced the net profit, followed by the final weight, dissolved oxygen concentration and temperature. The intensive photo-heterotrophic system is an innovative technology that allows to obtain high yields of shrimp production in a biosecure way. The economic results indicate a high potential for application and the convenience of continuing the investigation for its scaling up.

\section{Introducción}

La camaronicultura, a pesar de sus innegables beneficios como son la creación de empleos, la obtención de divisas para los países en desarrollo y de ganancias económicas para las empresas dedicadas a la actividad, es una industria que enfrenta grandes retos y problemáticas. Entre ellos, los de mayor importancia se relacionan con el impacto ambiental de los efluentes sobre los ecosistemas receptores de las descargas, la 
aparición frecuente de epizootias y la dependencia de insumos de origen animal, especialmente de peces marinos, para la fabricación de alimentos balanceados. Para enfrentar dichos retos, las nuevas tecnologías de cultivo requieren la sustentabilidad, tanto ambiental como económica, proporcionando organismos con alta calidad nutricional y organoléptica. La tendencia acuícola mundial busca la intensificación, ya que existe una competencia creciente por espacios y recursos con otras industrias, aunado a la necesidad de disminuir costos de producción para incrementar el margen de ganancia y la competitividad.

El sistema foto-heterotrófico es un sistema híbrido también llamado semi-biofloc y/o mixotrófico, que nace del sistema heterotrófico (biofloc) adaptando el protocolo a las características locales geográficas y de infraestructura. Está basado en un cuidadoso equilibrio entre organismos heterotróficos y autotróficos que se presenta cuando existe un balance en los nutrientes suministrados, la concentración de oxígeno disuelto adecuada y la capacidad de transporte de partículas necesaria, resultado del uso de aireación. Los microorganismos crean un agregado formado por algas verdes, bacterias, detritos, partículas orgánicas y protozoarios, que además de ser utilizado como alimento, limita el desarrollo de bacterias patógenas (ej. Vibrio spp.), controla la calidad del agua al degradar compuestos nitrogenados $\left(\mathrm{NH}_{4}, \mathrm{NO}_{3}, \mathrm{NO}_{2}\right)$, alimento no consumido, plancton muerto y heces fecales en compuestos no tóxicos, lo que conlleva a la estabilización, permitiendo que el recambio de agua sea innecesario si los demás parámetros son controlados correctamente (Huda et al., 2013). El objetivo del presente estudio fue evaluar el rendimiento biológico y económico de esta tecnología a través de un análisis bioeconómico.

\section{Materiales y métodos.}

Seis estanques de $1000 \mathrm{~m}^{2}(20 \times 50 \mathrm{~m})$ a una profundidad de $1.35 \mathrm{~m}$ fueron sembrados a una densidad de $120 \mathrm{PL} / \mathrm{m}^{2}$, alimentados 3 veces por día con ración comercial de $35 \%$ de proteína cruda. Rutinariamente se monitorearon y analizaron parámetros de calidad de agua (temperatura, salinidad, oxígeno disuelto, $\mathrm{pH}$, amonio, nitritos, nitratos, fosfatos, concentración de bacterias del género Vibrio y heterótrofas marinas). Aireadores (Aire- $\mathrm{O}_{2}$ ) de $2 \mathrm{HP}$ se utilizaron para ofrecer el equivalente a $20 \mathrm{HP} / \mathrm{ha}$ de aireación durante los primeros 60 días de cultivo, incrementando a $40 \mathrm{HP} /$ ha hasta finalizar el ciclo en el día 92. La salinidad se mantuvo en $46 \pm 2.5 \mathrm{~g} / \mathrm{L}$ con adiciones semanales de agua marina, equivalentes a $1.6 \%$ / día.

El modelo bioeconómico está integrado por tres submodelos: biológico, de manejo (factor de conversión alimenticia) y económico. El modelo fue calibrado a partir de los resultados zootécnicos y de calidad de agua de los estanques registrados a lo largo del ciclo. El submodelo biológico fue utilizado para predecir la cantidad de biomasa $\left(b_{t}\right)$ en función del tiempo, como el resultado del producto del peso individual promedio $\left(w_{t}\right)$ y el número de organismos sobrevivientes $\left(n_{t}\right): b_{t}=w_{t} . n_{t}$. Se usó la ecuación desarrollada por (Ruiz-Velazco et al., 2010) para predecir el crecimiento del camarón: $w_{t}=W_{i}+\left(W_{f-}\right.$ $\left.w_{i}\right)\left[\left(1-k_{t}\right) /\left(1-k^{h}\right)\right]^{3}+e$; dónde: $w_{t}$ es el peso promedio individual a $t$ unidades de tiempo, $w_{i}$ es el peso inicial, $w_{f}$ es el peso final, $k$ es el coeficiente de crecimiento, $h$ es el número de unidades de tiempo hasta la cosecha y e es el error residual.

La sobrevivencia fue calculada en función del tiempo, usando la ecuación exponencial: $n_{t}=n_{0} e^{-}$ $z t$; dónde: $n_{t}$ es el número de sobrevivientes, no es el número de organismos en la siembra, $t$ es el tiempo y $z$ es la tasa de mortalidad, calculada de la siguiente manera: $z=-L n\left(n_{f} / n_{0}\right) / t f$; dónde: $n_{f}$ es el número de organismos al momento de la cosecha (tf). Se usaron procedimientos para regresión no lineal disponibles en Statistica 6.0 para ajustar la ecuación de crecimiento, estableciendo un nivel de significación de $P<0.05$. Se llevaron a cabo análisis para determinar correlaciones entre los parámetros del submodelo biológico y las variables de calidad de agua.

Para el submodelo de manejo, el factor de conversión alimenticia (FCA) fue inferido en función del tiempo, combinando una ecuación lineal simple con una curva senoide, mediante la siguiente fórmula: $F C A=A+(B . t)+C{ }^{*} \operatorname{Sen}(2$. $\pi . t / L)+F+e$; dónde: $A$ y $B$ son coeficientes de la ecuación lineal, $C, D, L$ y $F$ son parámetros de la curva senoide, $t$ es el tiempo y e es el error residual resultante del análisis de regresión. En el caso del submodelo económico, la utilidad $\left(u_{t}\right)$ se calculó en función del tiempo: $u_{t}=i_{t}-c_{t}$; dónde: el ingreso $\left(i_{t}\right)$ es el producto de la biomasa del camarón (del submodelo biológico) y el precio de venta del camarón ( $p v)$ y $c_{t}$ son los costos operativos considerados para el análisis: $c_{t}=c p e r$ $+c P L+c f e r+c p e+c m a+c c+c A_{t}+c p_{t}+c r a_{t}+$ $c e_{t}$; dónde los costos son: cper, personal (incluyendo costos administrativos); $c P L$, postlarva; cfer, fertilizante; cpe, preparación del estanque; cma, mantenimiento; $c c$, cosecha; cat, alimento; $c p_{t}$, probióticos; $c r a t$, reemplazo de agua y $c_{t}$ energía eléctrica.

La normalidad en los datos de crecimiento de los estanques se analizó con la prueba de Kolmogorov-Smirnov y se comparó con la prueba análisis de varianza de una vía. El modelo bioeconómico se programó en Excel 10.0 y para los análisis se utilizó Statistica 6.0 (Statsoft, Tulsa, OK, USA), estableciendo una significación de $P<0.05$. Se llevaron a cabo simulaciones Monte Carlo con @Risk 6.0 (Palisade, Ithaca, N.Y), a fin de generar variabilidad estocástica en los valores de la producción y de la utilidad neta utilizando las distribuciones de probabilidad para cada uno de los submodelos. El coeficiente de variación se usó como un índice de incertidumbre del desempeño 
económico. Para analizar la sensibilidad de las utilidades a factores de riesgo, se utilizaron valores de los coeficientes de regresión múltiple, los cuales relacionan la utilidad neta con los respectivos parámetros zootécnicos y económicos. De acuerdo con esto, un valor alto del coeficiente, indica un alto impacto (sensibilidad) de la variable o parámetro correspondiente en los ingresos netos. Para el análisis de sensibilidad se utilizaron los procedimientos disponibles en @Risk 6.0.

\section{Resultados}

La ecuación de crecimiento analizada se ajustó adecuadamente a los datos previamente registrados y se obtuvieron resultados significativos en términos del análisis de varianza de la regresión (Fig. 1). Se estimó que el peso individual final ( $w f$ ) debería estar entre 16.72 y $19.16 \mathrm{~g}$, con un promedio de $18.14 \mathrm{~g}$. Con el mismo porcentaje de confianza se estimó que la sobrevivencia debería estar entre 80.3 y $83.9 \%$, promediando $81.4 \%$ y una biomasa final entre 16.81 y 18.59 Ton/ha, con un promedio de 17.73 Ton/ha, valores muy cercanos a los registrados en la base de datos (18.6 g para el peso final, $81.2 \%$ para la sobrevivencia y 18.1 Ton/ha para la biomasa final).

Los resultados mostraron que el coeficiente de crecimiento $(k)$ está negativamente relacionado con la temperatura del agua $(T)\left(r^{2}=0.93\right)$ y que el peso final ( $w f)$ está directamente relacionado con la concentración de oxígeno disuelto $(O D)$ $\left(r_{2}=0.92\right)$ y con la tasa de mortalidad $(z)\left(r^{2}=\right.$ $0.92)$. Las relaciones establecidas entre ellos son: $k=1.3139-0.0105(T)$ y $w f=1.0891+(3321.069$ $\cdot z)+(2.2626 \cdot D O)$. La ecuación resultante para el cálculo del factor de conversión alimenticia fue: $F C A=0.9209+(0.0077 \cdot t)-0.0413 \cdot \operatorname{Sen}(2 \cdot \pi \cdot t /$ $38.2618)+6.9397$.

La distribución de probabilidad de la utilidad se muestra en la Fig.2, se estimó que se obtendrá una utilidad entre $\$ 261,900$ y $\$ 1,129,800$ pesos $/$ ha $($ promedio $=\$ 693,730$ pesos $/$ ha $)$.

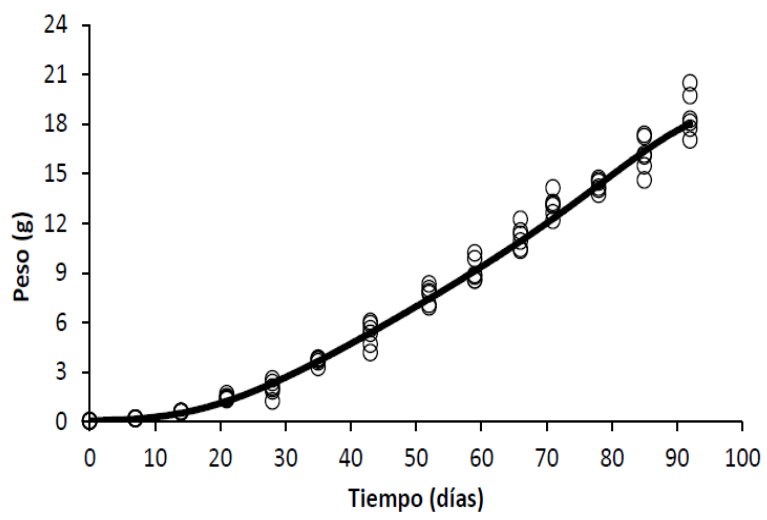

Fig.1. Ajuste de la ecuación de crecimiento a los valores reales

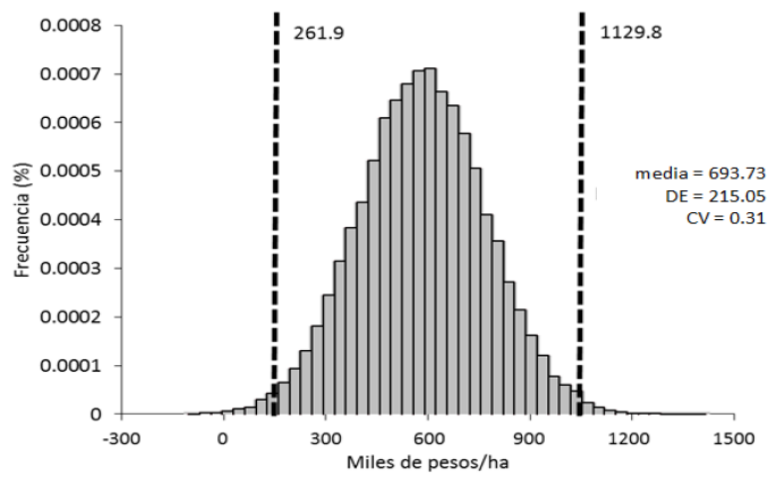

Fig.2. Distribución de probabilidad de la utilidad neta

De acuerdo con el análisis, los precios del camarón para las tallas de cosecha fueron los factores que más influyeron en la variabilidad de la utilidad, mientras que los parámetros de producción, de calidad del agua y el costo de la postlarva tuvieron influencia media o nula.

\section{Discusión y conclusión}

El modelo biológico predijo satisfactoriamente la dinámica de la producción del camarón, como consecuencia del ajuste adecuado de las ecuaciones de crecimiento y sobrevivencia, así como de los modelos estadísticos resultantes del análisis de regresión. Las relaciones entre los parámetros del modelo de producción y la calidad de agua fueron consistentes con lo reportado por 
otros autores en lo relativo a la influencia que tiene la temperatura y el oxígeno disuelto en el crecimiento y sobrevivencia. El peso final del camarón $\left(w_{f}\right)$ estuvo positivamente relacionado con la tasa de mortandad ( $z$ ) y la concentración de oxígeno disuelto $(O D)$. Una mayor tasa de mortandad significó una menor concentración de organismos, lo que facilitó un mayor crecimiento individual de los sobrevivientes, al haber mayor disponibilidad de espacio y recursos. El resultado del modelo estocástico mostró que, con el paso del tiempo, el coeficiente de variación disminuyó indicando que hay menor incertidumbre al predecir el valor de la biomasa hacia el final del ciclo.

Las granjas semi-intensivas en México, generan utilidades entre $\$ 13000$ y $\$ 45000$ pesos/ha/ciclo, mientras que para esta investigación, se estimó que la utilidad promedio fue de $\$ 693$ 730/ha. A pesar de los beneficios en bioseguridad y en la reducción de requerimientos de área productiva, son necesarias futuras investigaciones para demostrar completamente la factibilidad del sistema, tomando en cuenta, costos de inversión e impuestos, entre otras variables. El análisis de sensibilidad mostró que el precio del camarón fue el factor más importante en la variabilidad de la utilidad neta, en estudios anteriores, se indicó que es un factor muy importante en la determinación del rendimiento económico en granjas semiintensivas e intensivas (Hernández-Llamas et al. 2013, Estrada-Pérez et al. 2015). El precio base del camarón es principalmente determinado por comerciantes que acaparan la mayor parte de la producción y la mejor estrategia para la cosecha, posiblemente deberá cambiar año con año, dependiendo de cambios en los precios determinados por la demanda de tallas específicas y la aparición de brotes de enfermedades, entre otros factores.

La relevancia del precio del camarón para diseñar programas $y / 0$ protocolos de cultivo, hace imperativo el seguimiento y registros semanales en los precios del camarón, en vez de los actuales que se realizan mensual o trimestralmente. El modelo bioeconómico resultó una herramienta efectiva para analizar el rendimiento económico y factores de incertidumbre en los sistemas de cultivo de camarón.

\section{Referencias}

Estrada-Pérez, M., Ruiz-Velazco, J. M. J., Hernández-Llamas, A., Zavala-Leal, I. and Martínez-Cárdenas, L. (2015). Deterministic and stochastic models for analysis of partial harvesting strategies and improvement of intensive commercial production of white leg shrimp (Litopenaeus vannamei). Aquacultural Engineering, 70: 56-62. https://doi.org/10.1016/j.aquaeng.2015.11.003

Hernández-Llamas, A., Ruiz-Velazco, J. M. J. and Gómez-Muñoz, V. M. (2013). Economic risk associated with white-spot disease and stochastic variability in economic, zootechnical and water quality parameters for intensive production of Litopenaeus vannamei. Reviews in Aquaculture, 5: 121-131. https://doi.org/10.1111/raq.12008

Huda, A. S., Ispinanto, J., Bahri, F. and Decamp, O. (2013). Successful production in semi-biofloc in Indonesia. Aquaculture Asia Pacific, 2: 8-12. Disponible en: https://thefishsite.com/articles/successfulproduction-in-semibiofloc-in-indonesia

Ruiz-Velazco, J. M. J., Hernández-Llamas, A., Gómez-Muñoz, V. M. and Magallón, F. J. (2010). Dynamics of intensive production of shrimp Litopenaeus vannamei affected by White-spot disease. Aquaculture, 300: 113-119. https://doi.org/10.1016/i.aquaculture.2009.12.027 